\title{
NILPOTENT GLOBAL CENTERS OF LINEAR SYSTEMS WITH CUBIC HOMOGENEOUS NONLINEARITIES
}

\author{
JOHANNA D. GARCÍA-SALDAÑA, JAUME LLIBRE AND CLAUDIA VALLS
}

\begin{abstract}
In this paper we characterize the global nilpotent centers of polynomial differential systems of the form linear plus cubic homogeneous terms.
\end{abstract}

\section{Introduction AND STATEMENTS OF THE MAIN RESUltS}

Poincaré and Dulac in $[18,13]$ defined a center for a real planar vector field as a singular point whose neighborhood is filled of periodic orbits with the exception of the singular point. The so-called focus-center problem, which consists in distinguishing when a monodromic singular point is a focus or a center, started with them but it is still very active with many open problems (see for instance [2, 9]).

If a real planar analytic system has a center at the origin, then after a linear change of variables and a rescaling of its independent variable, it can be written in one of the following three forms:

$$
\dot{x}=-y+P(x, y), \quad \dot{y}=x+Q(x, y),
$$

called a non-degenerate center;

$$
\dot{x}=y+P(x, y), \quad \dot{y}=Q(x, y),
$$

called a nilpotent center;

$$
\dot{x}=P(x, y), \quad \dot{y}=Q(x, y),
$$

called a degenerate center, where $P(x, y)$ and $Q(x, y)$ are real analytic functions without constant and linear terms, defined in a neighborhood of the origin.

It is known (see $[19,6]$ ) that real planar quadratic polynomial differential systems have no global centers.

The global degenerated centers of homogeneous or quasi-homogeneous polynomial differential systems were characterized, respectively, in [8] and [17]. However the characterization of the global centers in the cases that the center is nilpotent or of linear-type with degree higher or equal than three has not been done. In [15] the authors provide, for the first time, a classification of the global linear-type centers for the systems having a linear part at the origin with purely imaginary eigenvalues and cubic homogeneous nonlinearities. However such a classification of the global centers is not known for the nilpotent centers, that is for the systems having a nilpotent singular point at the origin and cubic homogeneous nonlinearities. This classification is the main contribution of this paper.

Our first result is the following one.

Theorem 1. Any planar vector field of the form linear plus homogeneous cubic terms that at the origin of coordinates has a nilpotent singular point and at infinity has no singular

2010 Mathematics Subject Classification. 34C05, 34C07, 34C08.

Key words and phrases. nilpotent center, cubic polynomial differential system, global center, degenerated hyperbolic sector. 
points, after a linear change of variables and a rescaling of the variable independent can be written as either:

$$
\begin{aligned}
& \dot{x}=p_{1} x^{3}+\left(p_{2}-3 \alpha \mu\right) x^{2} y+p_{3} x y^{2}-\alpha y^{3}, \\
& \dot{y}=c x+\alpha x^{3}+p_{1} x^{2} y+\left(p_{2}+3 \alpha \mu\right) x y^{2}+p_{3} y^{3},
\end{aligned}
$$

or

$$
\begin{aligned}
& \dot{x}=a x+b y+p_{1} x^{3}+\left(p_{2}-3 \alpha \mu\right) x^{2} y+p_{3} x y^{2}-\alpha y^{3}, \\
& \dot{y}=-\left(a^{2} / b\right) x-a y+\alpha x^{3}+p_{1} x^{2} y+\left(p_{2}+3 \alpha \mu\right) x y^{2}+p_{3} y^{3},
\end{aligned}
$$

where $\alpha= \pm 1$ and $a, b, c, p_{1}, p_{2}, p_{3} \in \mathbb{R}$ with $\mu>-1 / 3$ and $b \neq 0$.

The proof of Theorem 1 is given is section 3 .

A singular point $p$ is called hyperbolic if the two eigenvalues of the Jacobian matrix at this point have nonzero real part. A singular point $p$ such that the determinant is zero and the trace of the Jacobian matrix at this point is different from zero is called semi-hyperbolic, and $p$ is isolated in the set of all singular points.

The singular points which are hyperbolic or semi-hyperbolic are called elementary. When the determinant and the trace are equal to zero but the Jacobian matrix at $p$ is not the zero matrix we say that $p$ is nilpotent.

Finally, if the Jacobian matrix at the singular point $p$ is identically zero and $p$ is isolated inside the set of all singular points then we say that $p$ is linearly zero, or degenerate. The study of its local phase portrait can be done with the directional blow-ups, see for more details $[3,7]$.

A polynomial differential system can be extended in a unique analytic way to infinity using the Poincaré compactification, for more details see Chapter 5 of [14].

Let $p$ be a singular point at infinity and let $h$ be a hyperbolic sector associated to $p$. We say that $h$ is degenerated if its two separatrices are contained in the equator of the Poincaré sphere. In the other case, $h$ is non-denegerated.

If a singular point $p$ at infinity is formed by two degenerated hyperbolic sectors then this point must be linearly zero. The previous statement follows taking into account that a singular point which is either hyperbolic (see Theorem 2.15 of [14]), or semi-hyperbolic (see Theorem 2.19 of [14]), or nilpotent (see Theorem 3.5 of [14]) cannot be formed by two degenerated hyperbolic sectors.

Theorem 2. Any planar vector field of the form linear plus homogeneous cubic terms that at the origin of coordinates has a nilpotent singular point and at infinity all the singular points in the Poincaré disc are linearly zero, after a linear change of variables and a rescaling of the independent variable, can be written as one of the following six systems:

$$
\begin{aligned}
\dot{x}=p_{1} x^{3}+p_{2} x^{2} y, & \dot{y}=c x+\alpha x^{3}+p_{1} x^{2} y+p_{2} x y^{2} ; \\
\dot{x}=a x+b y+p_{1} x^{3}+p_{2} x^{2} y, & \dot{y}=-\frac{a^{2}}{b} x-a y+\alpha x^{3}+p_{1} x^{2} y+p_{2} x y^{2} ; \\
\dot{x}=\left(p_{2}-3 \alpha\right) x^{2} y, & \dot{y}=c x+\left(p_{2}+3 \alpha\right) x y^{2} ; \\
\dot{x}=a x+b y+\left(p_{2}-3 \alpha\right) x^{2} y, & \dot{y}=-\frac{a^{2}}{b} x-a y+\left(p_{2}+3 \alpha\right) x y^{2} ; \\
\dot{x}=\left(p_{2}-3 \alpha\right) x^{2} y+p_{3} x y^{2}-\alpha y^{3}, & \dot{y}=c x+\left(p_{2}+3 \alpha\right) x y^{2}+p_{3} y^{3} ; \\
\dot{x}=a x+b y+\left(p_{2}-3 \alpha\right) x^{2} y+p_{3} x y^{2}-\alpha y^{3}, & \dot{y}=-\frac{a^{2}}{b} x-a y+\left(p_{2}+3 \alpha\right) x y^{2}+p_{3} y^{3},
\end{aligned}
$$

where $\alpha= \pm 1$ and $a, b, c, p_{1}, p_{2}, p_{3} \in \mathbb{R}$ with $b \neq 0$.

The proof of Theorem 2 is given in section 4 . 
Theorem 3. Systems (1) have a nilpotent center at the origin if and only if they can be written as one of the following two systems:

$$
\dot{x}=-3 \alpha \mu x^{2} y-\alpha y^{3}, \quad \dot{y}=c x+\alpha x^{3}+3 \alpha \mu x y^{2},
$$

where $c \in \mathbb{R}, \mu>-1 / 3, \alpha= \pm 1$ and $c \alpha>0$; and the system

$$
\dot{x}=\left(p_{2}-3 \alpha \mu\right) x^{2} y-\alpha y^{3}, \quad \dot{y}=c x+\alpha x^{3}+\left(p_{2}+3 \alpha \mu\right) x y^{2},
$$

where $c \in \mathbb{R}, p_{2} \neq 0, \mu>-1 / 3, \alpha= \pm 1$ and $c \alpha>0$.

Systems (9) are Hamiltonian with Hamiltonian function $H(x, y)=\frac{c}{2} x^{2}+\frac{3 \alpha \mu}{2} x^{2} y^{2}+\frac{\alpha}{4} x^{4}+$ $\frac{\alpha}{4} y^{4}$ and the center is global.

For systems $(10)$ the center is global if either $p_{2}^{2}-9 \mu^{2}+1 \geq 0$, or $p_{2}^{2}-9 \mu^{2}+1<0$ and $c\left(p_{2}-3 \alpha \mu\right)<0$.

Theorem 3 is proved in section 5 .

Theorem 4. Systems (2) have a nilpotent center at the origin if and only if they can be written as one of the following four systems:

$$
\begin{aligned}
& \dot{x}=a x+b y-3 \alpha \mu x^{2} y-\alpha y^{3}, \\
& \dot{y}=-\left(a^{2} / b\right) x-a y+\alpha x^{3}+3 \alpha \mu x y^{2},
\end{aligned}
$$

where $a, b \in \mathbb{R}, \mu>-1 / 3, \alpha= \pm 1$ and $b \alpha<0$ (systems (11) are Hamiltonian with Hamiltonian function $\left.H(x, y)=\frac{\alpha}{4} x^{4}+\frac{\alpha}{4} y^{4}-\frac{a^{2}}{2 b} x^{2}-\frac{b}{2} y^{2}-a x y+\frac{3}{2} \alpha \mu x^{2} y^{2}\right)$;

$$
\begin{aligned}
& \dot{x}=a x+a y+p_{1} x^{3}-3 \alpha \mu x^{2} y-p_{1} x y^{2}-\alpha y^{3}, \\
& \dot{y}=-a x-a y+\alpha x^{3}+p_{1} x^{2} y+3 \alpha \mu x y^{2}-p_{1} y^{3},
\end{aligned}
$$

where $a, p_{1} \in \mathbb{R}, p_{1} \neq 0, \mu>-1 / 3, \alpha= \pm 1$ and $a \alpha<0$;

$$
\begin{aligned}
& \dot{x}=a x-a y+p_{1} x^{3}-3 \alpha \mu x^{2} y-p_{1} x y^{2}-\alpha y^{3}, \\
& \dot{y}=a x-a y+\alpha x^{3}+p_{1} x^{2} y+3 \alpha \mu x y^{2}-p_{1} y^{3},
\end{aligned}
$$

where $a, p_{1} \in \mathbb{R}, p_{1} \neq 0, \mu>-1 / 3, \alpha= \pm 1$ and $a \alpha>0$;

$$
\begin{aligned}
& \dot{x}=a x+b y+p_{1} x^{3}-\left(\frac{a^{2}-b^{2}}{a b} p_{1}+\alpha\right) x^{2} y-p_{1} x y^{2}-\alpha y^{3}, \\
& \dot{y}=-\left(a^{2} / b\right) x-a y+\alpha x^{3}+p_{1} x^{2} y-\left(\frac{a^{2}-b^{2}}{a b} p_{1}-\alpha\right) x y^{2}-p_{1} y^{3},
\end{aligned}
$$

where $a, b, p_{1} \in \mathbb{R}, p_{1} \neq 0, a b \neq 0, \alpha= \pm 1$ and $b \alpha<0$.

The proof of Theorem 4 is given in section 6 .

Theorem 5. Under the assumptions of Theorem 4 the following statements hold.

(I) System (11) has a global center at the origin.

(II) System (12) has a global center at the origin if and only if one of the following sets of conditions hold:

(II.1) $\alpha=-1, \quad a>0, p_{1} \geq 3(1-\mu) / 4, \mu>-1 / 3, \quad b>0$;

(II.2) $\alpha=1, a<0, p_{1} \leq 3(\mu-1) / 4, \mu>-1 / 3, \quad b<0$.

(III) System (13) has a global center at the origin if and only if one of the following sets of conditions hold:

(III.1) $\alpha=-1, a<0, p_{1} \leq 3(\mu-1) / 4, \mu>-1 / 3, \quad b>0$;

(III.2) $\alpha=1, a>0, p_{1} \geq 3(1-\mu) / 4, \mu>-1 / 3, \quad b<0$.

(IV) System (14) has a global center at the origin.

The proof of Theorem 5 is given in section 7 .

Theorem 6. Systems (4) have a nilpotent center at the origin and no more finite singular points with all infinite singular points formed by two degenerated hyperbolic sectors (and 
consequently the center global) if and only if they can be written as one of the following two systems:

$$
\dot{x}=a x+b y, \quad \dot{y}=-\frac{a^{2}}{b} x-a y+\alpha x^{3},
$$

with $a, b \in \mathbb{R}, b \neq 0, \alpha= \pm 1$, and $b \alpha<0$ (system (15) is Hamiltonian with Hamiltonian function $\left.H(x, y)=\frac{\alpha}{4} x^{4}-\frac{a^{2}}{2 b} x^{2}-a x y-\frac{b}{2} y^{2}\right) ;$ and the system

$$
\dot{x}=a x+b y+p_{1} x^{3}+\frac{b}{a} p_{1} x^{2} y, \quad \dot{y}=-\frac{a^{2}}{b} x-a y+p_{1} x^{2} y+\frac{b}{a} p_{1} x y^{2}+x^{3},
$$

with $a, b \in \mathbb{R}, b<0$, and $a p_{1}>0$.

The proof of Theorem 6 is given in section 8 .

Theorem 7. Systems (3) and (5) do not have a nilpotent center at the origin.

The proof of Theorem 7 is given in section 9 .

Theorem 8. Systems (6) have a nilpotent center at the origin and no more finite singular points with all infinite singular points formed by two degenerated hyperbolic sectors if and only if they can be written as:

$$
\dot{x}=a x+b y-3 \alpha x^{2} y, \quad \dot{y}=-\left(a^{2} / b\right) x-a y+3 \alpha x y^{2},
$$

with $\alpha= \pm 1$ and $b \alpha>0$. The above system is Hamiltonian with Hamiltonian function

$$
H(x, y)=-\frac{a^{2}}{2 b} x^{2}-\frac{b}{2} y^{2}+\frac{3 \alpha}{2} x^{2} y^{2}-a x y .
$$

The proof of Theorem 8 is given in section 10 .

Theorem 9. Systems (7) have a nilpotent center at the origin and no more finite singular points with all infinite singular points formed by two degenerated hyperbolic sectors if and only if they can be written as one of the following two systems:

$$
\dot{x}=-3 \alpha x^{2} y-\alpha y^{3}, \quad \dot{y}=c x+3 \alpha x y^{2},
$$

with $c \in \mathbb{R}, \alpha= \pm 1$, and $c \alpha>0$ (system (18) is Hamiltonian with Hamiltonian function $\left.H(x, y)=\frac{c}{2} x^{2}+\frac{3 \alpha}{2} x^{2} y^{2}+\frac{\alpha}{4} y^{4}\right) ;$ and the system

$$
\dot{x}=\left(p_{2}-3 \alpha\right) x^{2} y-\alpha y^{3}, \quad \dot{y}=c x+\left(p_{2}+3 \alpha\right) x y^{2},
$$

with $\alpha= \pm 1, c \alpha>0$ and either $c\left(p_{2}+3 \alpha\right)>0$ or $c\left(p_{2}+3 \alpha\right) \leq 0$ and $p_{2}^{2} \geq 9$.

The proof of Theorem 9 is given in section 11 .

Theorem 10. Systems (8) have a nilpotent center at the origin and no more finite singular points with all infinite singular points formed by two degenerated hyperbolic sectors if and only if they can be written as:

$$
\dot{x}=a x+b y-3 \alpha x^{2} y-\alpha y^{3}, \quad \dot{y}=-\left(a^{2} / b\right) x-a y+3 \alpha x y^{2},
$$

with $\alpha= \pm 1, b \alpha<0$. System (20) is Hamiltonian with

$$
H(x, y)=\frac{a^{2}}{2 b} x^{2}-\frac{b}{2} y^{2}-a x y+\frac{3 \alpha}{2} x^{2} y^{2}+\frac{\alpha}{4} y^{4} .
$$

The proof of Theorem 10 is given in section 12 .

Corollary 11. Any polynomial vector field of the form linear plus cubic homogeneous terms having at the origin of coordinates a nilpotent singular point has a global center at the origin if and only if it satisfies the assumptions of Theorems 3, or, 5, or 6, or 7, or 8, or 9. 


\section{Classification of Cubic systems}

Following Theorem 3.2 given in [8], doing a linear change of variables and a rescaling of the independent variable, any planar cubic homogeneous system can be classified into the following ten classes:

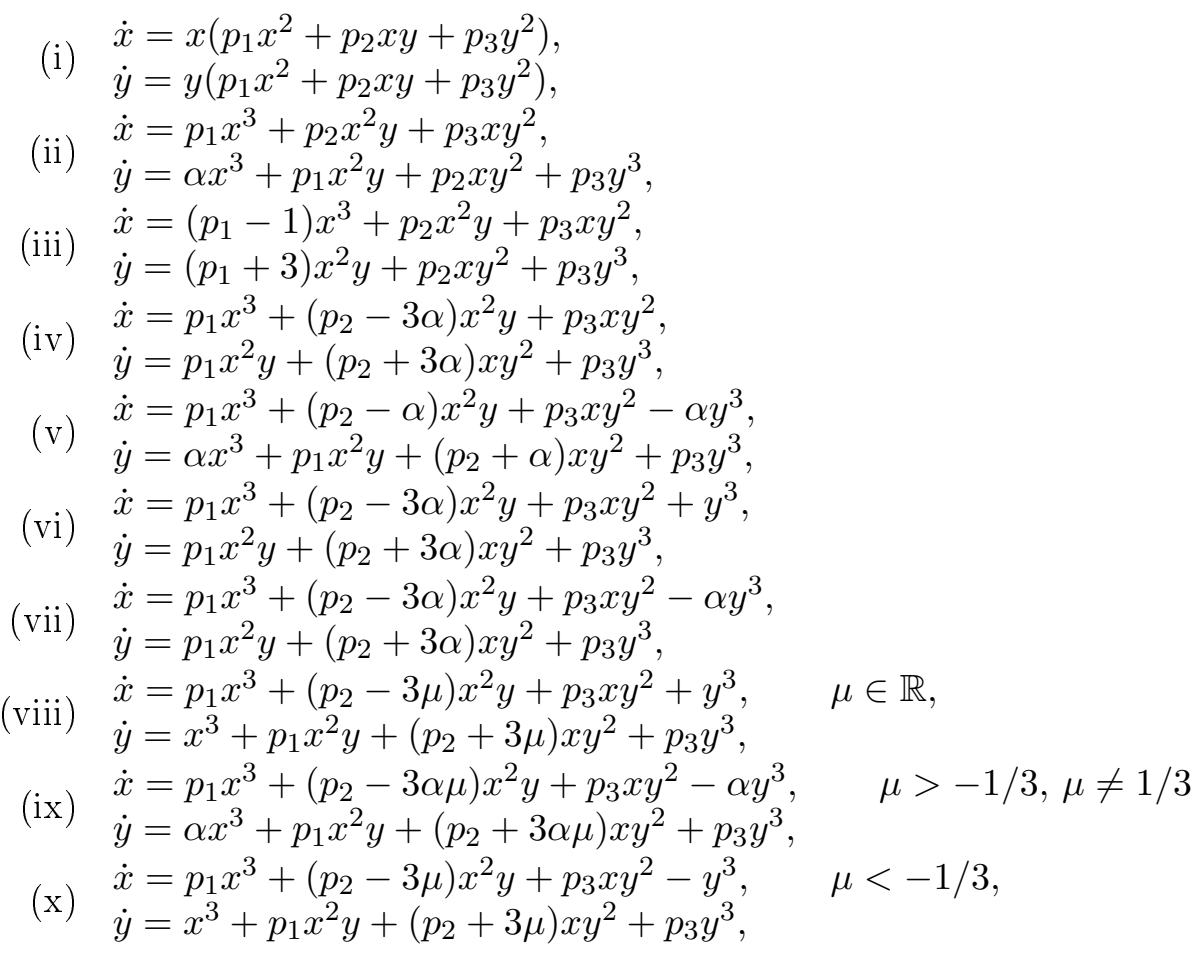

where $\alpha= \pm 1, p_{1}, p_{2}, p_{3} \in \mathbb{R}$.

\section{Proof of Theorem 1}

In section 2 is given the classification of planar cubic homogeneous differential systems. The behaviour at infinity of these ten families of systems in the Poincaré disc is as follows: the infinity of systems (i) is formed by singular points. The infinite singular points of systems (ii), (iii) and (iv) are the real solutions of $\alpha x^{4}=0,4 x^{3} y=0$, and $6 \alpha x^{2} y^{2}=0$ at infinity, respectively. System (v) has no singular points at infinity. The infinite singular points of systems (vi), (vii) and (viii) are the real solutions of $y^{2}\left(6 x^{2}-y^{2}\right)^{2}=0, \alpha y^{2}\left(6 x^{2}+y^{2}\right)=0$, and $x^{4}+6 \mu x^{2} y^{2}-y^{4}=0$ at infinity, respectively. System (ix) has no singular points at infinity. Finally, the infinite singular points of system (x) are the real solutions of $x^{4}+6 \mu x^{2} y^{2}+y^{4}=0$ at infinity.

Note that when $\mu=1 / 3$ system (v) becomes the (ix) system; that is why from now on, we consider system (ix) with $\mu>-1 / 3$ and forget system (v). In order to continue with the proof of the theorem, we will study systems (ix) with $\mu>-1 / 3$, because this family of systems is the only one that has no singular points at infinity.

On the other hand, for studying the cubic planar polynomial vector fields having linear and cubic terms being the origin a nilpotent point, it is sufficient to add to systems (i)-(x) a linear part. This is due to the fact that the linear changes of variables that are done to obtain systems (i)-(x) are not affine, they are strictly linear. So a linear plus a cubic vector field being the origin a nilpotent point with no infinite singular points in the Poincaré disc can be written as

$$
\begin{aligned}
& \dot{x}=a x+b y+p_{1} x^{3}+\left(p_{2}-3 \alpha \mu\right) x^{2} y+p_{3} x y^{2}-\alpha y^{3}, \\
& \dot{y}=c x+d y+\alpha x^{3}+p_{1} x^{2} y+\left(p_{2}+3 \alpha \mu\right) x y^{2}+p_{3} y^{3},
\end{aligned}
$$


where $a, b, c, d \in \mathbb{R}$ and $\alpha= \pm 1, \mu>-1 / 3$. The eigenvalues of the linear part of system (21) at the origin are

$$
\lambda_{1,2}=\frac{a+d \pm \sqrt{(a+d)^{2}-4(a d-b c)}}{2} .
$$

In order to have a nilpotent singular point, we need that $a=-d$ and the eigenvalues $\lambda_{1,2}= \pm \sqrt{a^{2}+b c} / 2$ must be equal to zero. So, if $b \neq 0$ we get $c=-\left(a^{2} / b\right)$, and if $b=0$ we have $a=0$ with $c \neq 0$ because the linear part of the system at the origin cannot be zero. Thus, the linear part of a planar polynomial vector field with a nilpotent singular point at the origin can be chosen to be either

$$
\dot{x}=0, \quad \dot{y}=c x,
$$

or

$$
\dot{x}=a x+b y, \quad \dot{y}=-\left(a^{2} / b\right) x-a y,
$$

where $a, b, c \in \mathbb{R}$ such that $b, c \neq 0$. Thus, system (21) becomes either system (1) or system (2). This completes the proof of the theorem.

\section{Proof of Theorem 2}

The proof of Theorem 2 is analogous to the proof of Theorem 1. It is done by considering the classification of cubic systems given in section 2 and taking into account the systems having singular points at infinity in the Poincaré disc that can be linearly zero.

System (ii) has a singular point at infinity: the origin of the local chart $U_{2}$. This point is linearly zero if and only if $p_{3}=0$. So, with system (ii) plus the linear part given by (22) we obtain system (3) and with system (ii) plus the linear part given by (23) we obtain system (4) of the statement of Theorem 2.

System (iv) has two singular points at infinity: the origin of the local chart $U_{1}$ and the origin of the local chart $U_{2}$. On $U_{1}$ the point is linearly zero if $p_{1}=0$, whereas on $U_{2}$ the origin is linearly zero if $p_{3}=0$. Thus, under these conditions system (iv) plus the linear part given by (22) provides system (5), and with system (iv) plus the linear part given by (23) yields system (6).

System (vii) has one singular point at infinity: the origin of the local chart $U_{1}$ which is linearly zero if and only if $p_{1}=0$. Thus, under this condition system (vii) plus the linear part given by (22) give place to system (7), and system (iv) plus the linear part given by (23) yields system (8).

Any of the other systems (i)-(x) do not meet the assumptions of the theorem, either because the infinity is formed by singular points, or because the system has no singular points at infinity, or if the system has singular points at infinity, at least one is not linearly zero.

\section{Proof of Theorem 3}

We will use the following result proved in [12] that allows to characterize the nilpotent centers for the differential systems $\dot{x}=y+P_{3}(x, y), \dot{y}=Q_{3}(x, y)$ which have a local analytic first integral, where $P_{3}$ and $Q_{3}$ are homogeneous polynomials of degree three.

Proposition 12. System

$$
\dot{x}=y+a_{21} x^{2} y+a_{12} x y^{2}+a_{03} y^{3}, \quad \dot{y}=-x^{3}+b_{21} x^{2} y+b_{12} x y^{2}+b_{03} y^{3},
$$

has a nilpotent center at the origin having a local analytic first integral if and only if one of the following two sets of conditions holds:

(a) $b_{21}=a_{12}+3 b_{03}=a_{21}+b_{12}=0$,

(b) $b_{21}=a_{12}=b_{03}=0$. 
To prove Theorem 3 first we do the change of variables $x=Y, y=X$ and the reparametrization of time $\tau=c t$ to system (1). By doing so we take the system to its real Jordan normal form, and we can apply Theorem 3.5 of [14] obtaining that

$$
Y=f(X)=-\left(p_{3} / c\right) X^{3}+\ldots,
$$

and then $B(X, f(X))=-(\alpha / c) X^{3}+\ldots$ and $G(X)=\left(4 p_{3} / c\right) X^{2}+\ldots$, therefore the origin of system (1) is a center or a focus if $c \alpha>0$.

In order to know when the origin is a center we apply Proposition 12 together with the results in $[1,4]$ which ensure that all the first integrals for these systems are analytic and not $C^{\infty}$ functions. To do that we shall write the system in the form of system (24), applying the change of variables $X=u-p_{3}(c \alpha)^{-1 / 2} v, Y=(\alpha / c)^{1 / 2} v$ and the reparametrization of time $s=(c \alpha)^{1 / 2} \tau$. Doing so we get the system

$$
u^{\prime}=v+a_{21} u^{2} v+a_{12} u v^{2}+a_{03} v^{3}, \quad v^{\prime}=-u^{3}+b_{21} u^{2} v+b_{12} u v^{2}+b_{03} v^{3},
$$

where the prime denotes derivative in the new variable $s$ and

$$
\begin{aligned}
& a_{21}=\frac{3 \mu+\alpha p_{2}+p_{3}^{2}}{\alpha c}, \quad a_{12}=\frac{p_{1}-\alpha p_{2} p_{3}-2 p_{3}^{3}-9 \mu p_{3}}{(\alpha c)^{3 / 2}}, \\
& a_{03}=\frac{1+p_{3}^{4}+6 \mu p_{3}^{2}}{c^{2}}, \quad b_{21}=\frac{4 p_{3}}{(\alpha c)^{1 / 2}}, \\
& b_{12}=\frac{-3 \mu+\alpha p_{2}-5 p_{3}^{2}}{\alpha c}, \quad b_{03}=\frac{p_{1}-\alpha p_{2} p_{3}+2 p_{3}^{3}+3 \mu p_{3}}{(\alpha c)^{3 / 2}} .
\end{aligned}
$$

Condition (a) of Proposition 12 gives $p_{1}=p_{2}=p_{3}=0$, and so we obtain system (9). Note that in this case system (9) is Hamiltonian with the Hamiltonian function given in the statement of the theorem. It was proved in [10] that the condition in order that the unique finite singular point is the origin is $c \alpha>0$. So the center of system (9) is global.

From condition (b) of Proposition 12 we obtain $p_{1}=p_{3}=0$. Thus, system (1) with $c \alpha>0$ has a nilpotent center at the origin if and only if $p_{1}=p_{3}=0$, and so we obtain system (3) of the statement of the theorem. Such a system has the following finite singular points: $(0,0),(0, \pm i \sqrt{c / \alpha})$, and

$$
\left( \pm i \sqrt{\frac{c\left(p_{2}-3 \alpha \mu\right)}{p_{2}^{2}-9 \mu^{2}+1}}, i \sqrt{\frac{c \alpha}{p_{2}^{2}-9 \mu^{2}+1}}\right),\left( \pm i \sqrt{\frac{c\left(p_{2}-3 \alpha \mu\right)}{p_{2}^{2}-9 \mu^{2}+1}},-i \sqrt{\frac{c \alpha}{p_{2}^{2}-9 \mu^{2}+1}}\right) .
$$

Note that due to condition $c \alpha>0$ the points $(0, \pm i \sqrt{c / \alpha})$ do not exist. In order that the other four points do not exist, the following conditions must hold: either $p_{2}^{2}-9 \mu^{2}+1 \geq 0$, or $p_{2}^{2}-9 \mu^{2}+1<0$ and $c\left(p_{2}-3 \alpha \mu\right)<0$. So, the theorem is proved.

\section{Proof of Theorem 4}

The idea of the proof is analogous to the proof of Theorem 3, so we will only explain the changes of variables and the most relevant part. First, we write the linear part of system (2) into its real Jordan normal form doing the change of variables $x=a X+Y, y=-\left(a^{2} / b\right) X$. Then we can apply Theorem 3.5 of [14] obtaining that the origin is a center or a focus if $b \alpha<0$. Now, to the resulting system we do the change of variables $X=u-k v, Y=v$ with

$$
k=\frac{a\left(a^{3} p_{3}-a^{2} b\left(p_{2}+3 \alpha \mu\right)+a b^{2} p_{1}-\alpha b^{3}\right)}{\left(-\alpha a^{2} b\left(a^{4}+6 \mu a^{2} b^{2}+b^{4}\right)\right)^{1 / 2}},
$$

and the rescaling $\tau=r t$ where

$$
r=\left(-\alpha a^{2}\left(a^{4}+6 \mu a^{2} b^{2}+b^{4}\right) / b^{3}\right)^{1 / 2}
$$

then the differential system becomes into the form of system (24). By applying Proposition 12 we have from condition (a) that the origin is a center if and only if $p_{1}=p_{2}=p_{3}=0$, 
and so we obtain system (11). Such a system is Hamiltonian with the Hamiltonian function given in the statement of the theorem.

Condition (b) of Proposition 12 yields the following real solutions in which $\alpha b<0$, $\alpha= \pm 1$ and $\mu>-1 / 3$ :

- $\mu=\frac{1}{3}$ and $p_{1}=p_{2}=p_{3}=0$. This condition is included in the previous case.

- $b=a, p_{2}=0$ and $p_{3}=-p_{1}$. Moreover, $p_{1} \neq 0$ because otherwise the system becomes system (11). This condition yields system (12) in the statement of the theorem;

- $b=-a, p_{2}=0$ and $p_{3}=-p_{1}$. Moreover, $p_{1} \neq 0$ because otherwise the system becomes system (11). This condition yields system (13) in the statement of the theorem;

- $\mu=\frac{1}{3}, p_{2}=-\frac{\left(a^{2}-b^{2}\right)}{a b} p_{1}, p_{3}=-p_{1}$ (and so $a b \neq 0$ ). Moreover, $p_{1} \neq 0$ because otherwise the system becomes system (11). This condition yields system (14) in the statement of the theorem.

This completes the proof of the theorem.

\section{Proof of Theorem 5}

It was proved in [10] that the Hamiltonian system (11) with the condition $b \alpha<0$ has only the origin as finite singular point. So statement $(I)$ is proved.

The singular points of system $(12)$ are $(0,0),\left( \pm \sqrt{\frac{2 a}{\alpha(1+3 \mu)}}, \pm \sqrt{\frac{2 a}{\alpha(1+3 \mu)}}\right),\left( \pm x_{+}, \mp y_{+}\right)$, $\left( \pm x_{-}, \mp y_{-}\right)$where

$$
x_{ \pm}=\sqrt{\frac{-a(\delta \pm \sqrt{-\alpha(1+3 \mu) \sigma})}{2 \gamma}}, \quad y_{ \pm}=\frac{\delta \mp \sqrt{-\alpha(1+3 \mu) \sigma}}{2\left(\alpha+p_{1}\right)} x_{ \pm},
$$

with $\delta=2 p_{1}+\alpha(1-3 \mu), \sigma=4 p_{1}+3 \alpha(1-\mu)$, and $\gamma=2 p_{1}^{2}-1+3 \mu$. Note that if $p_{1}=-\alpha$ or $\gamma=0$ then the singular points $\left( \pm x_{+}, \mp y_{+}\right),\left( \pm x_{-}, \mp y_{-}\right)$do not exist. So in order that the candidates to be singular points different from the origin do not exist we must have

$$
\alpha a<0 \text { and either } \alpha \sigma>0 \text {, or } \alpha \sigma \leq 0 \text { and } a \gamma(\delta \pm \sqrt{-\alpha(1+3 \mu) \sigma}) \geq 0 \text {. }
$$

Moreover, the above conditions are equivalent to the set of conditions given in statement $(I I)$ of the theorem. So statement $(I I)$ of the theorem is proved.

The singular points of system $(13)$ are $(0,0),\left( \pm \sqrt{\frac{2 a}{-\alpha(1+3 \mu)}}, \mp \sqrt{\frac{2 a}{-\alpha(1+3 \mu)}}\right),\left( \pm x_{+}, \pm y_{+}\right)$, $\left( \pm x_{-}, \pm y_{-}\right)$where

$$
x_{ \pm}=\sqrt{\frac{-a(\bar{\delta} \pm \sqrt{\alpha(1+3 \mu) \bar{\sigma}})}{2 \gamma}}, \quad y_{ \pm}=\frac{\bar{\delta} \mp \sqrt{\alpha(1+3 \mu) \bar{\sigma}}}{2\left(p_{1}-\alpha\right)} x_{ \pm},
$$

with $\bar{\delta}=2 p_{1}-\alpha(1-3 \mu), \bar{\sigma}=4 p_{1}-3 \alpha(1-\mu)$, and again $\gamma=2 p_{1}^{2}-1+3 \mu$. Note that if $p_{1}=\alpha$ or $\gamma=0$ then the singular points $\left( \pm x_{+}, \pm y_{+}\right),\left( \pm x_{-}, \pm y_{-}\right)$do not exist. So in order that the candidates to be singular points different from the origin do not exist we must have $\alpha a>0$ and either $\alpha \bar{\sigma}<0$ or $\alpha \bar{\sigma} \geq 0$ and $a \gamma(\bar{\delta} \pm \sqrt{\alpha(1+3 \mu) \bar{\sigma}}) \geq 0$. These conditions are equivalent to the set of conditions given in the statement $(I I I)$ of the theorem, so this completes the proof.

The singular points of system $(14)$ are $(0,0),( \pm a / \sqrt{b \alpha}, \pm \sqrt{b / \alpha}),\left( \pm x_{+}, \mp y_{+}\right),\left( \pm x_{-}, \mp y_{-}\right)$, where

$$
x_{ \pm}=\frac{\omega_{ \pm}}{\left(a^{2}+b^{2}\right) p_{1}}, \quad y_{ \pm}=\frac{\sqrt{-\alpha a b \rho} \mp a b p_{1}}{\left(a^{2}+b^{2}\right) p_{1}} \omega_{ \pm}
$$


with $\omega_{ \pm}=\sqrt{\alpha a^{4} b-a b^{2} \rho \pm 2 a^{2} b \sqrt{-\alpha a b \rho}}$ and $\rho=\left(a^{2}+b^{2}\right) p_{1}+\alpha a b$. So in order that the candidates to be singular points different from the origin do not exist we must have either $\alpha a b \rho>0$, or $\alpha a b \rho \leq 0$ and $\alpha a^{4} b-a b^{2} \rho \pm 2 a^{2} b \sqrt{-\alpha a b \rho} \leq 0$. Because of the conditions given in Theorem 4 for system (14) the above inequalities always hold, so the center is global. This completes the proof of the statement (IV) and concludes the proof of the theorem.

\section{Proof of Theorem 6}

First we write system (4) into its real Jordan normal form by doing the change of variables $x=a X+Y, y=-\left(a^{2} / b\right) X$. Then by applying Theorem 3.5 of [14] we have that the point is a center or a focus if $b \alpha<0$. Doing the change of variables $X=u-k v, Y=v$ where $k=\left(p_{2} a^{2}-p_{1} a b+\alpha b^{2}\right) /\left(-\alpha b^{3}\right)^{1 / 2}$ and the rescaling $\tau=a(-\alpha b)^{1 / 2} t$ to the system obtained previously we have a new system given in the form of system (24) with

$$
\begin{aligned}
& a_{21}=\frac{a^{2}\left(\alpha b^{2} p_{2}-\left(b p_{1}-a p_{2}\right)^{2}\right)}{\alpha b^{3}}, \quad a_{12}=\frac{a^{3}\left(a p_{2}-b p_{1}\right)\left(2\left(b p_{1}-a p_{2}\right)^{2}-\alpha b^{2} p_{2}\right)}{\left(-\alpha b^{9}\right)^{1 / 2}}, \\
& a_{03}=\frac{a^{4}\left(b p_{1}-a p_{2}\right)^{4}}{b^{6}}, \quad b_{12}=\frac{a^{2}\left(5\left(b p_{1}-a p_{2}\right)^{2}+\alpha b^{2} p_{2}\right)}{\alpha b^{3}}, \\
& b_{03}=-\frac{a^{3}\left(a p_{2}-b p_{1}\right)\left(2\left(b p_{1}-a p_{2}\right)^{2}+\alpha b^{2} p_{2}\right)}{\left(-\alpha b^{9}\right)^{1 / 2}}, \quad b_{21}=\frac{4 a\left(a p_{2}-b p_{1}\right)}{\left(-\alpha b^{3}\right)^{1 / 2}} .
\end{aligned}
$$

Condition (a) of Proposition 12 yields $p_{1}=p_{2}=0$ and so we obtain system (15). Such a system is Hamiltonian with the Hamiltonian function given in the statement of the Theorem. It was proved in [10] that system (15) with the condition $b \alpha<0$ has only the origin as finite singular point and at infinity only the origin of the local chart $U_{2}$ is a singular point and it has two degenerated hyperbolic sectors.

Condition (b) of Proposition 12 yields:

- $p_{1}=p_{2}=0$ which gives system (15).

- $p_{2}=(b / a) p_{1}$ with $a \neq 0$ and $p_{1} \neq 0$ because otherwise this system becomes (15).

System (4) with the condition $p_{2}=(b / a) p_{1}$ becomes

$$
\dot{x}=a x+b y+p_{1} x^{3}+\frac{b}{a} p_{1} x^{2} y, \quad \dot{y}=-\frac{a^{2}}{b} x-a y+\alpha x^{3}+p_{1} x^{2} y+\frac{b}{a} p_{1} x y^{2} .
$$

The above system does not have any additional finite singular point because of the condition $b \alpha<0$. Now, we will study the infinite singular points of system (25).

We already know that the unique infinite singular point is the origin of the local chart $U_{2}$ which is linearly zero so we need to apply the blow-up technique. The expression of system $(25)$ on the local chart $U_{2}$ is

$$
\dot{u}=b v^{2}+2 a u v^{2}+\frac{a^{2}}{b} u^{2} v^{2}-\alpha u^{4}, \quad \dot{v}=-\frac{b}{a} p_{1} u v-p_{1} u^{2} v+a v^{3}+\frac{a^{2}}{b} u v^{3}-\alpha u^{3} v .
$$

The singular direction in this case is $u=0$ so doing the directional blow-up $(u, v) \rightarrow(u, w)$ with $w=v / u$ and after removing the common factor $u$ we obtain the system

$$
\dot{u}=u\left(-\alpha u^{2}+b w^{2}+2 a u w^{2}+\frac{a^{2}}{b} u^{2} w^{2}\right), \quad \dot{v}=-w\left(\frac{b}{a} p_{1}+p_{1} u+b w^{2}+a u w^{2}\right) .
$$

When $u=0$ the singular points of the above system are $(0,0)$, and $\left(0, \pm \sqrt{-p_{1} / a}\right)$. The point $(0,0)$ is a semi-hyperbolic singular point, for applying Theorem 2.19 of [14] we need to have the condition $a b p_{1}<0$ and in this case the point is a saddle if $\alpha=1$. In order to have two hyperbolic sectors at the origin of the local chart $U_{2}$ we need that the singular points $\left(0, \pm \sqrt{-p_{1} / a}\right)$ do not exist, so the condition must be $a p_{1}>0$. Thus we obtain system (16) and the proof is complete. 


\section{Proof of Theorem 7}

We will only prove Theorem 7 for system (3) since the proof for system (5) follows in an analogous way. Doing the change of variables $x=Y, y=X$ and the reparametrization of time $\tau=c t$ system (3) is written in its real Jordan form as follows

$$
\dot{x}=y+c p_{2} x^{2} y+p_{1} x y^{2}+(\alpha / c) y^{3} \quad \dot{y}=c p_{2} x y^{2}+p_{1} y^{3} .
$$

By applying Theorem 3.5 of [14] we get that the origin of system (3) can not be a center (because $Y=f(X)=0, F(x)=B(X, f(X)) \equiv 0$ and $G(X) \equiv 0)$. This completes the proof.

\section{Proof of Theorem 8}

First we write system (6) into its real Jordan normal form by doing the change of variables $x=a X+Y, y=-\left(a^{2} / b\right) X$. Then by applying Theorem 3.5 of [14] we have that the point is a center or a focus if $b \alpha<0$. Doing the change of variables

$$
X=u-\frac{a\left(p_{2}+3 \alpha\right)}{(-6 \alpha b)^{1 / 2}} v, \quad Y=v,
$$

and the rescaling $\tau=-a^{2}(-6 \alpha / b)^{1 / 2} t$ to the system obtained previously we have a new system given in the form of system (24) with

$$
\begin{array}{ll}
a_{21}=-\frac{a^{2}\left(p_{2}^{2}-9\right)}{6 \alpha b}, & a_{12}=\frac{a^{3} p_{2}\left(p_{2}^{2}-9\right)}{3\left(-6 \alpha b^{3}\right)^{1 / 2}}, \\
a_{03}=\frac{a^{4}\left(p_{2}^{2}-9\right)^{2}}{36 b^{2}}, & b_{21}=\frac{2 \sqrt{2} a p_{2}}{(-3 \alpha b)^{1 / 2}}, \\
b_{12}=\frac{a^{2}\left(5 p_{2}^{2}-9\right)}{6 \alpha b}, & b_{03}=-\frac{a^{3} p_{2}\left(p_{2}^{2}-9\right)}{3\left(-6 \alpha b^{3}\right)^{1 / 2}} .
\end{array}
$$

Statement (a) and (b) of Proposition 12 yield the same conditions. That is,

- $a=0$, this condition yields a quadratic system, so it is not interesting for us.

- $p_{2}=0$ and $a \neq 0$, then we obtain

$$
\dot{x}=a x+b y-3 \alpha x^{2} y, \quad \dot{y}=-\frac{a^{2}}{b} x-a y+3 \alpha x y^{2} .
$$

The above system is Hamiltonian with the Hamiltonian function given in the statement of the theorem. The singular points of this system are

$$
(0,0), \quad\left( \pm \sqrt{\frac{2 b}{3 \alpha}}, \pm \sqrt{\frac{2 a}{3 b \alpha}}\right) .
$$

Because of the condition $\alpha b<0$, only the origin is a finite singular point of system (27). Moreover, we already know that the origins of the local charts $U_{1}$ and $U_{2}$ are the infinite singular points and they are linearly zero so we need to apply the blow-up technique. The expression of system (27) on the local chart $U_{1}$ is

$$
\dot{u}=6 \alpha u^{2}-\frac{a^{2}}{b} v^{2}-2 a u v^{2}-b u^{2} v^{2}, \quad \dot{v}=3 \alpha u v-a v^{3}-b u v^{3} .
$$

Doing the $u$-directional blow-up $(u, v) \rightarrow(u, w)$ with $w=v / u$ and after removing the common factor $u$ we obtain the system

$$
\dot{u}=6 \alpha u-\frac{a^{2}}{b} u w^{2}-2 a u^{2} w^{2}-b u^{3} w^{2}, \quad \dot{v}=-3 \alpha w+\frac{a^{2}}{b} w^{3}+a u w^{3} .
$$


When $u=0$ the real singular point of the above system is $(0,0)$. The eigenvalues of the Jacobian matrix at this point are $6 \alpha$ and $-3 \alpha$ so the point is a saddle. Doing the directional blow-up $(u, v) \rightarrow(w, v)$ with $w=u / v$ and after cancel the common factor $v$ we obtain

$$
\dot{u}=-\frac{a^{2}}{b}-a v w+3 \alpha w^{2}, \quad \dot{v}=-a v^{2}-b v^{3} w+3 \alpha v w .
$$

On $v=0$ the above system does not have singular points. Then after the blow-down the origin of the local chart $U_{1}$ is formed by two degenerated hyperbolic sectors.

The expression of system (27) on the local chart $U_{2}$ is

$$
\dot{u}=-6 \alpha u^{2}+b v^{2}+2 a u v^{2}+\frac{a^{2}}{b} u^{2} v^{2}, \quad \dot{v}=-3 \alpha u v+a v^{3}+\frac{3 a^{2}}{b} u v^{3} .
$$

The only singular direction is $u=0$. So doing the directional blow-up $(u, v) \rightarrow(u, w)$ with $w=v / u$ and after removing the common factor $u$ we obtain the system

$$
\dot{u}=-6 \alpha u+b u w^{2}+2 a u^{2} w^{2}+\frac{a^{2}}{b} u^{3} w^{2}, \quad \dot{v}=3 \alpha w-a u w^{3}-b w^{3} .
$$

Since we have the condition $\alpha b<0$, the only singular point of the above system on $u=0$ is $(0,0)$. The eigenvalues of the Jacobian matrix at this point are $-6 \alpha$ and $3 \alpha$ so the point is a saddle. Then after the blow-down the origin of the local chart $U_{2}$ is formed by two degenerated hyperbolic sectors. Thus we obtain system (17) and the proof is complete.

\section{Proof of Theorem 9}

First we write system (7) into its real Jordan normal form by doing the change of variables $x=Y, y=X$ and the rescaling of the time $\tau=c t$. Then by applying Theorem 3.5 of [14] we have that the point is a center or a focus if $\alpha c>0$. Doing the change of variables $X=u-p_{3}(\alpha c)^{-1 / 2} v, Y=(\alpha / c)^{1 / 2} v$ and the rescaling $t=(\alpha / c)^{1 / 2} \tau$ to the system obtained previously we have a new system given in the form of system (24) with

$$
\begin{aligned}
& a_{21}=\frac{3+\alpha p_{2}+p_{3}^{2}}{\alpha c}, \quad a_{12}=-\frac{p_{3}\left(9+\alpha p_{2}+2 p_{3}^{2}\right)}{(\alpha c)^{3 / 2}}, \\
& a_{03}=\frac{p_{3}^{2}\left(6+p_{3}^{2}\right)}{c^{2}}, \quad b_{12}=\frac{4 p_{3}}{(\alpha c)^{1 / 2}}, \\
& b_{03}=\frac{-3+\alpha p_{2}-5 p_{3}^{2}}{\alpha c}, \quad b_{21}=\frac{p_{3}\left(3-\alpha p_{2}+2 p_{3}^{2}\right)}{(\alpha c)^{3 / 2}} .
\end{aligned}
$$

Condition (a) of Proposition 12 yields $p_{2}=p_{3}=0$ and so we obtain system (18). Such a system is Hamiltonian with the Hamiltonian function given in the statement of the theorem. It was proved in [10] that system (18) with the condition $\alpha c>0$ has only the origin as finite singular point and at infinity only the origin of the chart $U_{2}$ is singular point and it is formed by two degenerated hyperbolic sectors.

Condition (b) of Proposition 12 yields the conditions: $p_{3}=0\left(p_{2} \neq 0\right.$ because otherwise this system becomes system (18)). System (7) with the condition $p_{3}=0$ becomes

$$
\dot{x}=\left(p_{2}-3 \alpha\right) x^{2} y-\alpha y^{3}, \quad \dot{y}=c x+\left(p_{2}+3 \alpha\right) x y^{2} .
$$

So system (30) with the condition $\alpha c>0$ has a nilpotent center at the origin. The singular points of the above system are

$$
(0,0), \quad\left( \pm i \sqrt{\frac{c}{p_{2}+3 \alpha}}, \pm \sqrt{\frac{\alpha c}{9-p_{2}^{2}}}\right) .
$$

Note that if $p_{2}=-3 \alpha$ then only the origin is a singular point. Taking into account that $\alpha c>0$, to have only the origin as singular point we must have either $c\left(p_{2}+3 \alpha\right)>0$ or $c\left(p_{2}+3 \alpha\right) \leq 0$ and $p_{2}^{2} \geq 9$. 
Now we study the behavior of singular points at infinity of (30) and obtain the conditions for which these singular points have two degenerate hyperbolic sectors. We already know that the origin of the local chart $U_{1}$ is a singular point and it is linearly zero. The expression of system (30) on the local chart $U_{1}$ is

$$
\dot{u}=c v^{2}+6 \alpha u^{2}+\alpha u^{4}, \quad \dot{v}=-\left(p_{2}-3 \alpha\right) u v+\alpha u^{3} v .
$$

The singular points of the above system are

$$
(0,0), \quad\left( \pm \sqrt{\frac{p_{2}-3 \alpha}{\alpha}}, \pm \sqrt{\frac{9-p_{2}^{2}}{\alpha c}}\right) .
$$

Note that on $v=0$ the origin is the only singular point of system (31) and it is linearly zero, so to know its behavior we need to apply the blow-up technique. The characteristic polynomial is $-v\left(c v^{2}+\left(p_{2}+3 \alpha\right) u^{2}\right)$ so we only have the characteristic direction $v=0$. Doing the $u$ directional blow-up $(u, v) \mapsto(u, w)$ with $w=v / u$, and removing the common factor $u$ between $\dot{u}$ and $\dot{w}$ we obtain the system

$$
\dot{u}=6 \alpha u+c u w^{2}+\alpha u^{3}, \quad \dot{w}=-\left(p_{2}+3 \alpha\right) w-c w^{3} .
$$

When $u=0$, the only singular point of (32) is the origin and its eigenvalues are $-\left(p_{2}+3 \alpha\right)$ and $6 \alpha$ then the point is a saddle because of the conditions $c \alpha>0$ and $c\left(p_{2}+3 \alpha\right)>0$. After the blow-down the origin of the local chart $U_{1}$ is formed by two degenerated hyperbolic sectors. In short, in order to have only the origin as the finite singular point of system (30), and the origin of the local chart $U_{1}$ formed by two degenerate hyperbolic sectors, we must have $\alpha c>0$ and $c\left(p_{2}+3 \alpha\right)>0$. Hence we obtain system (19). Therefore the proof of the theorem is complete.

\section{Proof of Theorem 10}

We write (8) into its real Jordan normal form by doing the change of variables $x=a X+Y$, $y=-\left(a^{2} / b\right) X$. Then by applying Theorem 3.5 of [14] we have that the origin is a center or a focus if $b \alpha<0$. Doing the change of variables

$$
X=u-\frac{a^{3}\left(a p_{3}-\left(p_{2}+3 \alpha\right) b\right)}{\left(-\alpha a^{4} b\left(a^{2}+6 b^{2}\right)\right)^{1 / 2}} v, \quad Y=v,
$$

and the rescaling $\tau=\left(-b^{3} /\left(\alpha a^{4}\left(a^{2}+6 b^{2}\right)\right)\right)^{1 / 2} t$ to the system obtained previously we obtain a new system given in the form of system (24), so we can apply Proposition 12.

Condition (b) of Proposition 12 is empty and condition (a) yields $p_{2}=p_{3}=0$. So system (8) becomes

$$
\dot{x}=a x+b y+-3 \alpha x^{2} y-\alpha y^{3}, \quad \dot{y}=-\frac{a^{2}}{b} x-a y+3 \alpha x y^{2} .
$$

The above system is Hamiltonian with the Hamiltonian function given in the statement of the theorem. At infinity, system (33) with the condition $\alpha b<0$, has only the origin of the chart $U_{1}$ as singular point and it is formed by two degenerated hyperbolic sectors, see [10].

The finite singular points of system $(33)$ are $(0,0)$ and

$$
\begin{aligned}
& p_{2,3}= \pm\left(\frac{(3 b+\sqrt{S})}{12 a} \sqrt{\frac{2(R-b \sqrt{S})}{3 \alpha b}}, \sqrt{\frac{R-b \sqrt{S}}{6 \alpha b}}\right), \\
& p_{4,5}= \pm\left(\frac{(3 b-\sqrt{S})}{12 a} \sqrt{\frac{2(R+b \sqrt{S})}{3 \alpha b}}, \sqrt{\frac{R+b \sqrt{S}}{6 \alpha b}}\right),
\end{aligned}
$$

where $R=2 a^{2}+3 b^{2}$ and $S=-12 a^{2}+9 b^{2}$. If $S<0$, then the singular points $p_{2,3}$ and $p_{4,5}$ are not real. If $S \geq 0$ then $R \pm b \sqrt{S}>0$, so the singular points in this case are not real. So the finite singular point is only the origin. This completes the proof of Theorem 10 . 


\section{ACKNOWLEDGEMENTS}

J.D. García-Saldaña is supported by FONDECyT grant 11171115/2017. J. Llibre is supported by the Ministerio de Economía, Industria y Competitividad - Agencia Estatal de Investigación grant MTM2016-77278-P (FEDER), the Agència de Gestió d'Ajuts Universitaris i de Recerca grant 2017 SGR 1617, and the European project Dynamics- H2020MSCA-RISE-2017-777911, and C. Valls is partially supported by FCT/Portugal through UID/MAT/04459/2013.

\section{REFERENCES}

[1] A. Algaba, C. García, J. Giné and J. Llibre, The center problem for $\mathbb{Z}_{2-\text { symmetric nilpotent }}$ vector fields, to appear in J. Math. Anal. Appl.

[2] A. Algaba, C. García and J. Giné, Nondegenerate centers and limit cycles of cubic Kolmogorov systems, Nonlinear Dynam. 91 (2018), 487-496.

[3] M.J. Álvarez, A. Ferragut and X. Jarque A survey on the blow up technique, Int. J. Bifurcation and Chaos 21 (2011), 3103-3118.

[4] A. F. Andreev, A.P.Sadovski and V.A. Tsikalyuk, The center-focus problem for a system with homogeneous nonlinearities in the case of zero eigenvalues of the linear part, (Russian) Differ. Uravn. 39 (2003), no. 2, 147-153, 285; translation in Differ. Equ. 39 (2003), no. 2, 155-164.

[5] A. A. Andronov, E. A. Leontovich, I. I. Gordon and A. G. Maier, Qualitative Theory of Second-Order Dynamical Systems, John Wiley \& Sons, New York, 1973.

[6] J.C. Artés, J. Llibre and N. Vulpe, Complete geometric invariant study of two classes of quadratic systems, Electronic J. Differential Equations 2012 (2012), No.9, pp. 1-35.

[7] V. I. Arnold and Y. S. Ilyashenko, Dynamical Systems I, Ordinary Differential Equations. Encyclopaedia of Mathematical Sciences, Vol. 1-2, Springer-Verlag, Heidelberg, 1988.

[8] A. Cima and J. Llibre, Algebraic and topological classification of the homogeneous cubic vector fields in the plane, J. Math. Anal. and Appl. 147 (1990), 420-448.

[9] C. Christopher and C. Li, Limit cycles of differential equations, Advanced Courses in Mathematics, CRM Barcelona, Birkhäuser Verlag, Basel, 2007.

[10] I. Colak, J. Llibre and C. Valls Hamiltonian nilpotent center of linear plus cubic homogenous polynomial vector fields, Adv. Math., vol. 259, (2014) 655-687.

[11] I. Colak, J. Llibre and C. Valls Hamiltonian linear type centers of linear plus cubic homogeneous polynomial vector fields, J. Differential Equations, 257 (2014), 1623-1661.

[12] J. Chavarriga, H. Giacomini, J. Giné and J. Llibre Local analytic integrability for nilpotent centers, Ergod. Th. \& Dynam. Sys. 23 (2003), 417-428.

[13] H. Dulac, Détermination et integration d'une certaine classe d'équations différentielle ayant par point singulier un centre, Bull. Sci. Math. 32 (1908), 230-252.

[14] F. Dumortier, J. Llibre and J. C. Artés, Qualitative Theory of Planar Differential Systems, UniversiText, Springer-Verlag, New York, 2006.

[15] J. D. García-Saldaña, J. Llibre and C. Valls, Linear type global centers of linear systems with cubic homogeneous nonlinearities, preprint, 2018.

[16] E.A.V. Gonzales, Generic properties of polynomial vector fields at infinity, Trans. Amer. Math. Soc. 143 (1969), 201-222.

[17] W.P. Li, J. Llibre, J.Yang and Z. Zhang, Limit cycles bifurcating from the period annulus of quasihomogeneous centers, J. Dynam. Differential Equations 21 (2009), 133-152.

[18] H. Poincaré, Mémoire sur les courbes définies par les équations différentielles, Oeuvreus de Henri Poincaré, 1, Gauthier-Villars, Paris, 1951, pp. 95-114.

[19] N. Vulpe, Affine-invariant conditions for the topological discrimination of quadratic systems with a center, Differential Equations 19 (1983), 273-280.

J.D. García-Saldaña: Departamento de Matemática y Física Aplicadas, Universidad Católica de la Santísima Concepción, Alonso de Ribera 2850, Concepción, Chile

E-mail address: jgarcias@ucsc.cl

J. Llibre: Departament de Matemàtiques, Universitat Autònoma de Barcelona, 08193 Bellaterra, Barcelona, Spain

E-mail address: jllibre@mat.uab.es 
C. Valls: Departamento de Matemática, Instituto Superior Técnico, Universidade de LisBoa, Av. Rovisco Pais, 1049-001, Lisboa, Portugal

E-mail address: cvalls@math.ist.utl.pt 